\title{
Multi-node processing for asymmetrical communications in underwater acoustic networks
}

\author{
António Silva, Julien Huillery and Sérgio M. Jesus \\ Institute for Systems and Robotics, Universidade do Algarve \\ Campus de Gambelas, PT-8005-139 Faro, Portugal \\ Email: \{asilva,jhuillery,sjesus\}@ualg.pt
}

\begin{abstract}
Underwater acoustic communication networks have attracted attention for its applications in many areas such as mine warfare, rapid environmental assessment or search and rescue, where there is the need for conserted action of a group of observers/actuators. A common requirement in most of these applications is the need to make accessible to the global (terrestrial/aerial) user network large amounts of critical underwater collected data. This paper addresses this requirement by using multichannel nodes providing an asymmetrical point-topoint $(\mathbf{P} 2 \mathrm{P})$ connection where the upload link has a much higher data rate than the download link. The emphasis of this work is not so much on the final channel throughput but on establishing the optimal processing of spatially distributed multichannel nodes that serve as interface between the underwater nodes and the global/user network. The adopted strategy relies on passive time-reversal which can be viewed as a spatial pre-equalizer for each multichannel node. Then an intersymbol interference optimal combination of various nodes is divised for balancing poor communications of one or more nodes in the network. The method and techniques are theoretically derived and applied to real data acquired with a network of 2 surface buoys over an environmentally challenging area off the coast of Portugal in July 2007.
\end{abstract}

\section{INTRODUCTION}

In recent years underwater acoustic networks became one of the most challenging topics in ocean acoustics. Typically such networks comprise underwater nodes, fixed and mobile, that are simultaneously used to acquire environmental information, to communicate such information and to relay it from other nodes in a network fashion. Often the messaging between nodes comprises small pieces of information with the status of the actuators, time, position, speed and commands, which can be supported by low data rate and robust connections. Concurrent with this low speed messaging, situations often arise that require the transmission of high chunks of data as, for example, an optical or acoustic image, sonar audio file, oceanographic measurements, etc, which can not be handled by underwater nodes with poor power output and limited autonomy. In these cases the solution adopted in this work is to make available specifically designed nodes for interfacing the underwater network with the global/user network that asymmetrically handle commands down to the underwater network at low data rate and provides a high throughput upload data link from the underwater network to the global network as it is shown in figure 1. Depending on the application and operational scenario these nodes, herein designated as interface

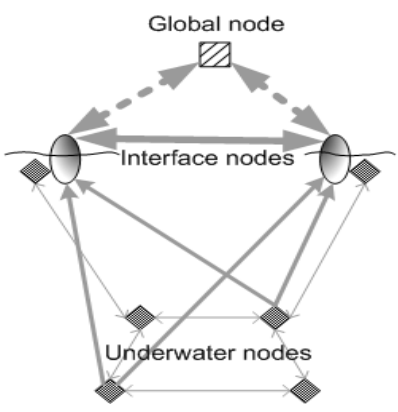

Fig. 1. Network configuration. The global node communicates with the interface nodes using a radio link. The interface nodes communicates with the underwater nodes using an acoustic non-coherent low data rate bidirectional link to send commands and receive status information, simultaneously uses a coherent high data rate uploading link to receive huge data form the underwater nodes. The underwater nodes communicate with each other using a bidirectional low data rate to send commands, to receive status information and to relay data from more distant nodes. The arrows thickness represents the data throughput of the connections.

nodes, may be mobile as for example carried on surface deployable buoys or fixed on land cabled platforms. While the later has no specific limitations the price paid for the mobility of the former is the power and processing limited capabilities. A typical scenario of a mobile communication network is shown in figure 2 where the red surface buoys with vertical line array (VLA) represent the multichannel interface nodes receiving $\mathrm{P} 2 \mathrm{P}$ upload connections from underwater nodes either bottom fixed or mobile (AUVs). The data received on the surface buoys can then be relayed to the user network through direct shore wireless/radio connection, satellite relay, ship or aerial stations. Depending on the problem at hand and operational situation, the multinode processing can normally be achieved on land, ship or aerial based stations.

In general, embarked/standalone underwater nodes are implemented with single transducer and secure non-coherent modems, whereas interface nodes use multichannel coherent systems that explore spatial diversity for decreasing channel intersymbol interference (ISI) and increasing signal-to-noise ratio (SNR). One of the most often overlooked situation in commercial underwater communication systems is when in practical cases, due to environmental adversity or any other unknown cause, communication becomes very difficult or even impossible. This is where multi-node/network processing 


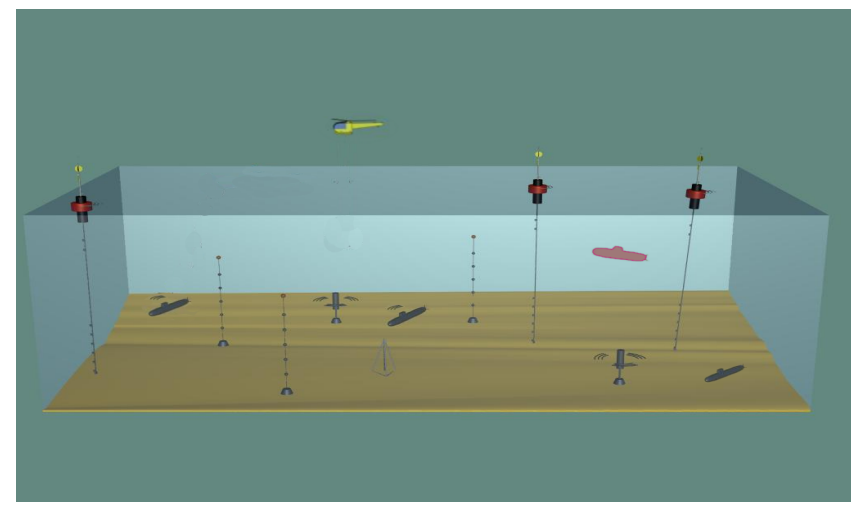

Fig. 2. Mobile communication network involves the integration of acoustic and non-acoustic systems for collecting and mobile nodes for uploading environmental information

makes sense as long as the spatial/environmental distribution is taken into account in the processing and system performance is optimized accordingly.

Spatial environmental diversity could be tackled almost at the hydrophone level e.g. by using the reciprocity property of the underwater acoustic channel in a method called Time Reversal (TR) [1], [2] that was shown to efficiently focalize a received signal, in time and space, at the source position in the presence of an unknown environment. In initially experiments, TR made use of a the VLA sensor outputs that collect the signal transmitted by the source and an array of transducers collocated with the VLA that retransmits a time reversed version of the received signals. Such experiments showed the capability of the acoustic channel to deconvolve itself which is an essential property in underwater communications. The same concept can be applied in a slightly different way using only one source and one VLA. In this case, before the transmission of the signal, the source must transmit a probe signal which is pulse compressed at the receiver so as to generate estimates of the channel Impulse Responses (IRs). The IRs estimates are then time-reversed and used as the backpropagation acoustic channels for data focusing [3]. Such technique is termed Passive Phase Conjugation (PPC) [4] in the frequency domain or passive TR (pTR) in the time domain. The ability of pTR to produce a time focus makes it attractive for underwater communications [3], [5]. In fact, the time focalization property of the pTR is equivalent to the deconvolution of the multipath introduced by the channel.

This paper explores the pTR capabilities to reduce the ISI at a single multichannel interface node and then proposes an ISI optimal technique to further reduce network communications dependence of environmental adversity by combining multiple interface nodes' outputs. The proposed technique will be termed Minimum ISI Multi-node Addition (MISIMA) and consists in an weighed addition of the nodes' outputs with the optimal weighs computed with a closed form expression for ISI reduction. Although MISIMA was derived using the pTR equalization it can be applied with any other coherent equalization technique at the node level. The proposed methods are derived theoretically and then applied on an experimental data set acquired during the RADAR'07 sea trial, off the coast of Portugal in July 2007. At a given moment during that experiment, BPSK communication signals in a $12.5 \mathrm{kHz}$ band were transmitted across a $400 \mathrm{~m}$ depth submarine canyon both to a $3.5 \mathrm{~km}$ away VLA suspended from a drifting Acoustic Oceanographic Buoy (AOB) at the border of the canyon and a second $\mathrm{AOB} / \mathrm{VLA}$ at $5.5 \mathrm{~km}$ range from the source and located further into the continental platform. It turned out that this complex bathymetry resulted in a different communication performance between the two AOB nodes which served to motivate and test the developed methods.

This paper is organized in the following manner. In section II the application of the pTR communication system concept to the AOBs network will be presented. In section III the experimental configuration will be described. In section IV the AOBs network communication system will be applied to real data. Finally, section V summarizes the main results, draw some conclusions and suggest future research.

\section{Network Passive Time Reversal}

This section setup the theoretical background for the implementation of a high data rate uploading link between one underwater node and the interface network. For the Point to Point (P2P) communication, the pTR processor is considered. Throughout the paper convolution is denoted by the binary operator $*$ and as usually done in the context of pass-band coherent communications, a complex representation in terms of baseband equivalent signals will de adopted.

Let us assume that the transmitted signal is Pulse Amplitude Modulated (PAM) written as

$$
s(t)=a(t) * p(t),
$$

where $p(t)$ is the symbols pulse shape and

$$
a(t)=\sum_{n=-\infty}^{+\infty} a_{n} \delta\left(t-n T_{b}\right),
$$

where $a_{n}$ is a zero mean symbol sequence assumed to be white with power $\sigma_{a}^{2}$, and $T_{b}$ is the symbol duration. After transmitting $s(t)$ from a single source over the underwater channels $h_{i, j}(t)$, where $i$ represents the hydrophone index of the $j^{\text {th }}$ VLA, and applying the pTR processor it results

$$
z_{j}(t)=y_{j}(t)+x_{j}(t)
$$

where $y_{j}(t)$ contains the desired data-signal contaminated with residual ISI and $x_{j}(t)$ the noise disturbances defined to be zero mean and white stochastic signal in the signal bandwidth as shown in [6]. The signal $y_{j}(t)$ is given by

$$
y_{j}(t)=s(t) * p_{T R, j}(t)
$$

where $g_{j}(t)$ is the global IR resulting from the propagation of the signal in the ocean and the application of the pTR processor

$$
g_{j}(t)=\sum_{i=1}^{I}\left[h_{i, j}^{*}(-t) * h_{i, j}^{\prime}(t)\right] .
$$


In the 'ideal' case where the assumptions associated with timereversal ${ }^{1}$ are fulfilled, $g_{j}(t)$ can be approximated by a dirac pulse if there is no environmental mismatch in the channel between the probe $\left(h_{i, j}(t)\right)$ and the data transmissions $\left(h_{i, j}^{\prime}(t)\right)$ IRs. In practice the TR basic principle associated assumptions are not fully accomplished, the channel stationarity is not achieve $h_{i, j}(t) \neq h_{i, j}^{\prime}(t)$, and the use of the probe channel estimate $h_{i, j}(t)$ as a FIR filter requires a previous time limitation [6]. All those contribute to degrade the dirac pulse approximation and to increase the residual ISI.

After sampled at the symbol rate, the pTR output $z_{j}(t=$ $\left.n T_{b}\right)$ contains the transmitted symbol sequence $a(n)$ contaminated with ISI. The contribution of the ISI is evaluated as the ratio between the power of the multipath spread of the pTR overall IR sampled at the symbol rate $\left(\left(g\left(t=n T_{b}\right)\right.\right.$ with $n \neq 0$ ) and its main path power (with $n=0$ ),

$$
I_{j}=\frac{\sum_{n \neq 0}\left|g_{j}^{2}(n)\right|}{\left|g_{j}^{2}(0)\right|}
$$

with $g_{j}(t)$ given by (4).

The pTR output sampled at the symbol rate, $z_{j}(n)$, is usually represented by a constellation that should be synchronized with the transmitted data symbols previously to slicing, that is symbols estimate. Such synchronization operation corresponds to rotate the pTR overall IR, $g_{j}(t)$, in order to make its main path $g_{j}(0)$ a positive real number. Henceforth, with no loss of generality, $g_{j}(0)$ will be considered a positive real number.

\section{A. Multi-node processing}

Figure 1 shows that the data transmitted from one underwater node will be received on all the interface nodes VLA, and processed with a local pTR demodulator resulting in an output $z_{j}(t)$ given by (3). It will be shown that by re-scaling the initial IRs $g_{j}(n)$ by $\alpha_{j}$, each ISI given by (5) keeps unchanged, but the global ISI of their weighed sum can be minimized by selecting the appropriate weighs $\alpha_{j}$. The optimum selection of the coefficients $\alpha_{j}$ is the main issue of the MISIMA system.

The MISIMA multi-node processing starts by (re)sampling the demodulator output to the symbol rate and synchronizing the constellations. Then making a weighed addition of the $z_{j}(n)$ output signals of all nodes it results

$$
z(n)=\sum_{j=1}^{J} \alpha_{j} z_{j}(n)=y(n)+x(n),
$$

where $x(n)$ is the resulting noise term given by

$$
x(n)=\sum_{j=1}^{J} \alpha_{j} x_{j}(n),
$$

where, since $x_{j}(n)$ are uncorrelated [6], it will result a Signal to Noise Ratio (SNR) enhancement in $z(n)$ given by (6). In

\footnotetext{
${ }^{1}$ i.e., there is a sufficiently large number of hydrophones, the vertical array is spanning the whole water column and the propagation environment is timeinvariant.
}

(6) $y(n)$ contains the desired signal contaminated with ISI, which according to (4) is given by

$$
y(n)=\left.s(t) * g(t)\right|_{t=n T_{b}},
$$

where $g(t)$ results from the weighed summation of the overall IRs of each pTR processor $g_{j}(t)$ and is given by

$$
g(t)=\sum_{j=1}^{J} \alpha_{j} g_{j}(t)
$$

that is the overall IR when considering the set of $J$ VLAs. The overall IRs of each pTR processor $g_{j}(t)$ can be approximated by a dirac pulse at lag zero (main arrival), contaminated with residual multipath at different lags. Since the VLAs are placed at different locations the multipath structures of one $g_{j}(t)$ will be uncorrelated with the multipath structure of all the others. Such spatial diversity suggests that the weighed addition (8) will result in a power enhancement of the main arrival at zero lag and in a destructive multipath combination at nonzero lags.

For the purpose of computing the ISI in the overall IR (8), with no loss of generality it will be considered only the presence of $J=2$ VLAs. Applying the ISI metric (5) in (8) it results

$$
I=\frac{\sum_{n \neq 0}\left|\left(\alpha_{1} g_{1}\left(n T_{b}\right)+\alpha_{2} g_{2}\left(n T_{b}\right)\right)^{2}\right|}{\left|\left(\alpha_{1} g_{1}(0)+\alpha_{2} g_{2}(0)\right)^{2}\right|} .
$$

that is the global ISI, of the overall IR when considering the set of $J$ VLAs. The global ISI (9) can be expressed as a function of each VLA/pTR overall IR, $I_{j}$, as

$$
\begin{array}{r}
I=\frac{I_{1}\left|\left(\alpha_{1} g_{1}(0)\right)^{2}\right|}{\left|\left(\alpha_{1} g_{1}(0)+\alpha_{2} g_{2}(0)\right)^{2}\right|}+\frac{I_{2}\left|\left(\alpha_{2} g_{2}(0)\right)^{2}\right|}{\left|\left(\alpha_{1} g_{1}(0)+\alpha_{2} g_{2}(0)\right)^{2}\right|}+ \\
\frac{2 \sum_{n \neq 0}\left\langle\alpha_{1} g_{1}(n), \alpha_{2} g_{2}(n)\right\rangle}{\left|\left(\alpha_{1} g_{1}(0)+\alpha_{2} g_{2}(0)\right)^{2}\right|}
\end{array}
$$

where the global ISI, $I$, depends on a weighed sum of the initials ISIs, $I_{1}$ and $I_{2}$, added by the normalized inner product summation, where $\left\langle g_{1}(n), g_{2}(n)\right\rangle$ represents the inner product of each $n$ complex element of the multipath.

The global ISI (10) can be minimized by making

$$
\frac{\alpha_{1}}{\alpha_{2}}=\frac{I_{2}\left|g_{2}^{2}(0)\right|\left|g_{1}(0)\right|-\left|g_{2}(0)\right| \sum_{n \neq 0}\left\langle g_{1}(n), g_{2}(n)\right\rangle}{I_{1}\left|g_{1}^{2}(0)\right|\left|g_{2}(0)\right|-\left|g_{1}(0)\right| \sum_{n \neq 0}\left\langle g_{1}(n), g_{2}(n)\right\rangle} \text {. }
$$

that gives a family of solutions that results from the constant ratio of $\alpha_{1}$ and $\alpha_{2}$. It is no obvious from (10) with $\alpha_{1}$ and $\alpha_{2}$ given by (11) that there is always an ISI attenuation due to the weighed addition (6). Nevertheless it can be shown that $I<\min \left(I_{1}, I_{2}\right)$ if

$$
\sum_{n \neq 0}\left|\frac{g_{1}(n)}{g_{1}(0)}-\frac{g_{2}(n)}{g_{2}(0)}\right|^{2}>0
$$

which always holds, reviling that in a stationary environment MISIMA always attenuate the ISI of the node with smaller ISI. Due to the underwater channel variability it is expected that the $\alpha_{j}$ coefficients loss validity and the MISIMA performance degrades. 
The proposed Minimum ISI Multi-node Addition (MISIMA) make use of a single coefficient per network VLA/pTR node to optimize the ISI of there weighed addition. In MISIMA the $\alpha_{j}$ coefficients are computed by a closed form expression (11) that use as inputs parameters that where taken from the equivalent IR, $g_{j}(n)$, of the pTR equalizer at each node. Since the equivalent IR is a common framework of any communication system the MISIMA system can be applied with other equalization techniques, at the interface node level, rather then the pTR equalizer.

\section{EXPERIMENTAL DESCRIPTION}

To illustrate the MISIMA multi-node processing described in section II, underwater communication data collected during the RADAR'07 experiment will be used. This experiment took place in the vicinity of the Setúbal canyon, in a site located approximately $50 \mathrm{~km}$ south of Lisbon (Portugal), from 10 to 15 July 2007. During the experiment, Binary Phase Shift Keying (BPSK) modulated sequences with a central frequency of $12.5 \mathrm{kHz}$ where transmitted at a symbol rate of 2000 baud and a roll-off factor of 0.5 from a single source. Before the data transmission an upward linear chirp was emitted as a probe signal for channel estimation. The emitted BPSK sequences were collected by two receivers (interface nodes in the network context) distant from approximately $2 \mathrm{kms}$ and consisting of a VLA of hydrophones attached to a surface-suspended and free-drifting the Oceanographic Acoustic Buoy (AOB). The AOB ( [7]) is a telemetry buoy that meets the traditional sonobuoys characteristics of small size and weight, but with the advanced characteristics of having a sufficiently dense array of hydrophones and thermistors, a self localizing GPS system, processing capabilities, a high throughput radio data link, and a large data storage capacity. The use of the AOBs network for environmental inversion has been shown in [8]-[10]. For the study reported in this paper, each node of the AOBs network make use o a pTR equalizer to increase the $\mathrm{P} 2 \mathrm{P}$ communications performance and their outputs are post-processed with the MISIMA to further increase the performance.

Thereafter, the VLA nodes will be named AOB1 and AOB2 respectively. The first VLA (AOB1) is composed of 8 hydrophones. The two first hydrophones are located at 10 and 15 meters depth respectively, while the 6 remaining hydrophones span the water column from 55 to 80 meters depth with a regular spacing of 5 meters. The second VLA (AOB2) consists of 16 hydrophones, 4 meters spaced, spanning the water column from 6 to 66 meters depth.

The acoustic source was towed by the research vessel NRC D.Carlos I, which slowly cruised in the direction of the two AOBs, from an initial distance of approximately 3.5 and 5.5 kms from AOB1 and AOB2, respectively. The source depth remained constant around 60 meters.

Together with the bathymetric characteristics of the site, figure 3 displays the GPS positions of the two free-drifting AOBs as well as the source ship track. The crosses indicate the locations of the three elements corresponding to the data

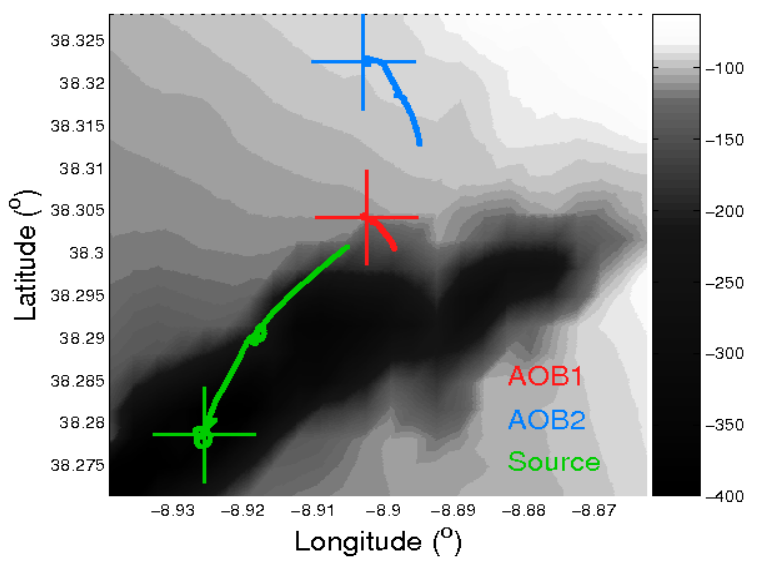

Fig. 3. Bathymetry of the experimental area, location of the two receivers AOB1 and AOB2, and displacement of the acoustic source during the RADAR'07 sea trial. For the data presented in section IV, the crosses indicate the position of the three elements.

presented in section IV. It should be noticed the rangedependent geometry of the experiment. An important geological depression, the Setúbal canyon, is present underneath the source. At the time of the transmission reported in the next section, the watercolumn at the location of the source is around 400 meters. On the other side, the two AOBs were located upon the continental plateau in a slowly varying water depth region, ranging from 90 meters for AOB2 to 120 meters for AOB1.

The network considered in this work thus consists of $J=2$ nodes interfacing the underwater and surface environments. In the next section, the increase of communication performance obtained by combining the equalized sequences from the two nodes is illustrated and quantified.

\section{Real Data Multi-Node Processing Results}

The pTR processor requires the knowledge of the propagation channels between the source and each hydrophone. The channels IRs were estimated by pulse compression, i.e. the correlation of the received signals with an emitted probe. In the RADAR'07 experiment, an upward linear frequency modulation centered on the carrier frequency $(12.5 \mathrm{kHz})$ and with a bandwidth of a $7 \mathrm{kHz}$ was used. The obtained IRs or arriving patterns are displayed in figure 4-a) for AOB1 and figure 4-b) for AOB2. At the edge of the Setúbal canyon, AOB1 is located in a complex environment for underwater sound propagation with several multipath generated on the borders of the canyon. As a result, largely delayed multipath effects and strong reverberation are observable on the arriving pattern of AOB1. The situation of AOB2 appears less complex as the different arrivals are more clearly resolved. For both AOB1 and $\mathrm{AOB} 2$, the white dashed lines represent the beginning and the end of the IRs considered in the pTR equalizer [6]. As they are more stationary, only the first arrivals have been keeped to represent the "backpropagation" channels. 
(a)

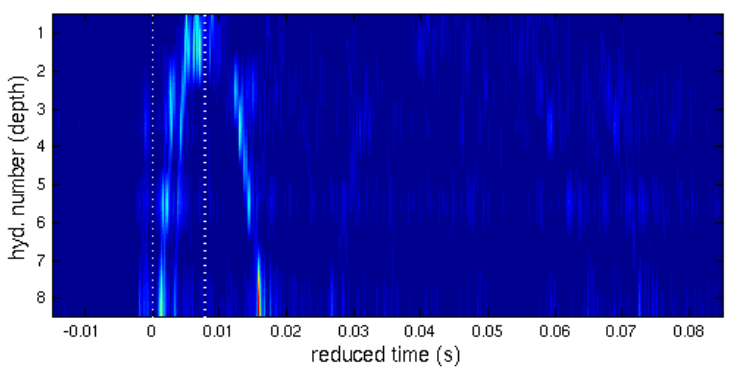

(b)

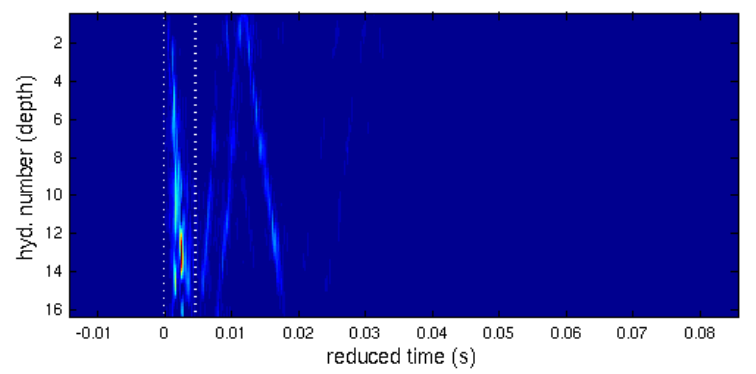

Fig. 4. Arriving pattern obtained by pulse compression of the upward linear chirp probe signal received at (a) AOB1 and (b) AOB2. The thick dashed white lines represent the beginning and the end of the impulse responses considered in the pTR equalizer.

Figure 5-a) displays the ISI (in [dB]), for the IR estimates of 16 communication sequences, for the following cases: applying (5) in (4) for AOB1 alone (dashed blue line), and AOB2 alone (dash-dotted green line); applying (9) in (8) for Multi-node Addition (MA) with $\alpha_{1}=\alpha_{2}=1$ (plain red line) and MISIMA with $\alpha_{1}$ and $\alpha_{2}$ given by (11) (plain cyan line). To reduce the number of hydrophones differences between the two nodes only the first 8 hydrophones of AOB2, thereafter named АОВ2a, where used in figure 5-b) that shows the behavior of the same four cases when considering AOB1 and AOB2a.

We first emphasize the different performances of AOB1 (figure 5-a,b), dashed blue line), AOB2 (figure 5-a), dash-dotted green line) and AOB2a (figure 5-b), dash-dotted green line). For the 16 communication sequences the mean ISI are -2.69 $\mathrm{dB},-10.57 \mathrm{~dB}$ and $-5.46 \mathrm{~dB}$ for AOB1, AOB2, AOB2a respectively. Two reasons can be advanced to explain their strong difference. First, the number of hydrophones attached with AOB2 is twice as great as with AOB1 and AOB2a. Second, as already discussed, AOB1 rests in an adverse location for acoustic communication, whereas the environment faced by $\mathrm{AOB} 2$ and $\mathrm{AOB} 2 \mathrm{a}$ is much more favorable.

In this context for the combination of $\mathrm{AOB} 1$ and $\mathrm{AOB} 2$, MA (figure 5-a), plain red line) and MISIMA (figure 5-a), plain cyan line) it results that for all 16 IR estimates the mean ISI are $-11.25 \mathrm{~dB}$ and $-11.44 \mathrm{~dB}$ respectively. For the combination of $\mathrm{AOB} 1$ and $\mathrm{AOB} 2 \mathrm{a}$ figure 5-b) shows that the mean ISI are $-6.42 \mathrm{~dB}$ and $-6.74 \mathrm{~dB}$ for MA and MISIMA respectively. (a)

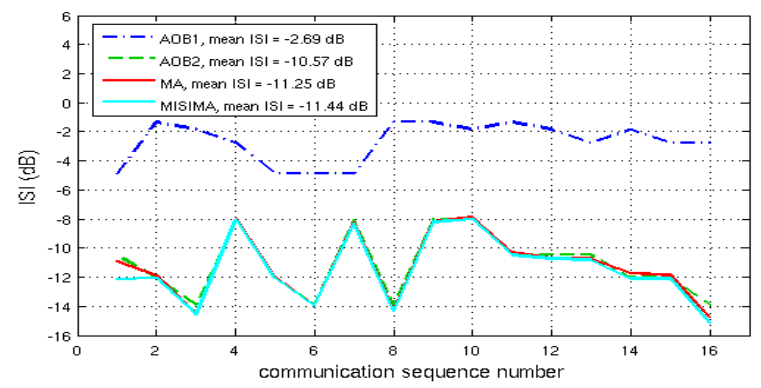

(b)

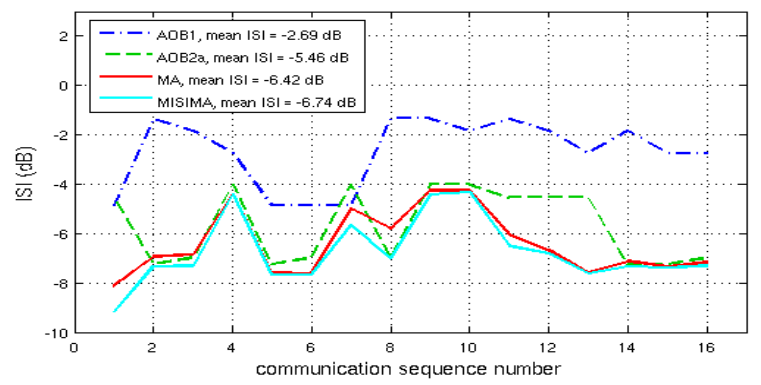

Fig. 5. ISI (in $\mathrm{dB}$ ) when processing: (a) AOB1 with 8 hydrophones and AOB2 with 16 hydrophones;(b) AOB1 with 8 hydrophones and AOB2a with 8 hydrophones. pTR equalizer outputs for AOB1 (dashed blue line), AOB2 (dash-dotted green line), the MA addition of both (plain red line) and MISIMA addition (plain light-blue line).

Along the 16 communication sequences figure 5-a,b) shows that MA occasionally presents a poor performance then the best of the AOBs and that MISIMA always present a better performance than all the others. Namely in figure 5-b) it can be observed The MISIMA ISI reduction is higher when the AOBs ISI are of the same order. It is thus likely to note that the MISIMA multi-node processing performances do not degrade as one of the node presents severe difficulties, preserving the efficiency of the network in an 'at least equal to the best' manner.

The results reported in figure 5 assumes stationarity since are only based on the application of MISIMA to the probe signal IR estimates. Figure 6 displays the Mean Squared Error (MSE) between the transmitted BPSK sequence and the demodulated sequence during 10 seconds obtained for the first of the 16 communication sequences of figure 5. During those 10 seconds there will be an environmental variability mainly due to the source-AOB relative range and depth variations but also due to other environmental parameters changes as surface waves, etc.

Figure 6-a) displays the MSE (in $[\mathrm{dB}]$ ) for the following cases: for AOB1 alone (dashed blue line), and AOB2 alone (dash-dotted green line) considering their outputs given by (3); for MA with $\alpha_{1}=\alpha_{2}=1$ (plain red line) and MISIMA with $\alpha_{1}$ and $\alpha_{2}$ given by (11) (plain cyan line) considering their outputs given by (6). Figure 6-b) shows the behavior of the same four cases when AOB2 with 16 hydrophones is replaced by AOB2a with 8 hydrophones. The mean results for the 10 
(a)

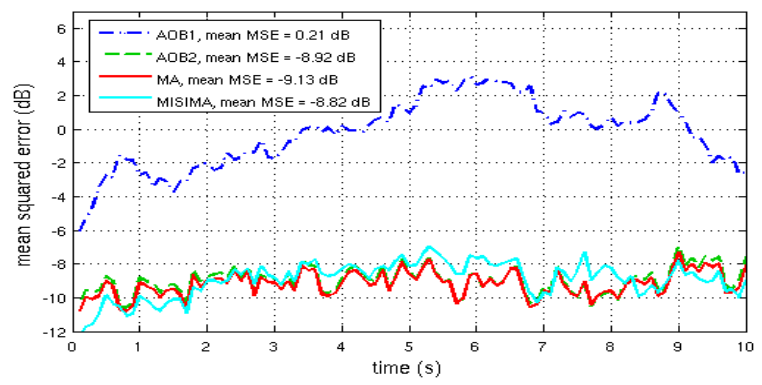

(b)

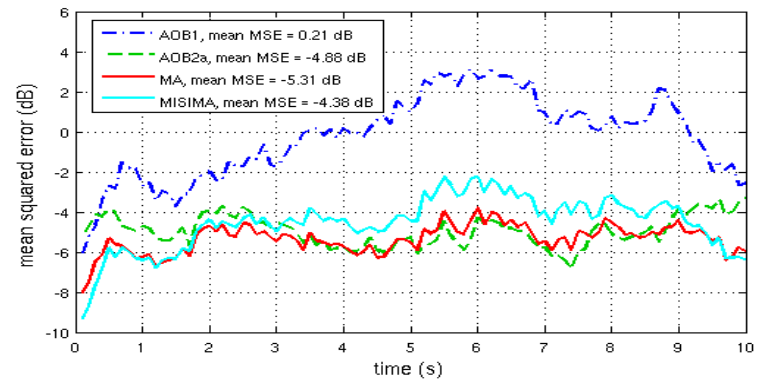

Fig. 6. MSE (in $\mathrm{dB}$ ) for 10 seconds of data obtained for the communication sequence number 1 of figure 5 . The same cases as those of figure 5 are plotted.

seconds of data in figure 6-a) and (b) shows that the MA with an MSE of $-9.13 \mathrm{~dB}-5.31 \mathrm{~dB}$ respectively (corresponding to Bit Error Rate (BER) of $0 \%$ and $1.71 \%$ ) presents the best performance. Nevertheless when considering only the first 2 seconds of data of figure 6-a) and (b) the best performance is achieved by MISIMA with a MSE of $-10.53 \mathrm{~dB}$ (and $0 \%$ $\mathrm{BER}$ ) and $-6.32 \mathrm{~dB}$ (and $0.94 \% \mathrm{BER}$ ) respectively.

Considering the closed relation between ISI and MSE [11] and comparing the ISI results for the first of the 16 communication sequences of figure 5 with the initial values for the MSE of figure 6 it can be observed that they are quite similar. Since the results of figure 6 where obtained with a probe signal that was transmitted 0.3 seconds before the data such similarity reveals that in presence of a short time environmental mismatch the pTR in AOB1, AOB2 and AOB2a, their simple addition in MA and the $\alpha_{j}$ coefficients in MISIMA preserve their ISI minimization characteristics. As the elapsed time between the probe and data transmission increases the AOB1 performance degrades substantially wile AOB2 and AOB2a MSE is almost constant, revealing that despite the AOBs are placed only $2 \mathrm{~km}$ apart, the location of AOB1 presents a much higher environmental variability. Comparing the MA and the MISIMA curves in figure 6 it can be seen that MA is less sensitive to the performance degradation of AOB1 than MISIMA. This suggest that the MISIMA coefficients should be made adaptive in order to accommodate for the ISI and $g_{j}(0)$ variations which result from the channel IRs variability.

\section{CONClusion And Future Work}

The work presented in this paper focuses on the improvement of a high data rate uploading communication link in an underwater acoustic network. It is examined how the coherent combination of BPSK sequences demodulated at several nodes can improve the overall communication performances.

It is first shown that, due to the spatial diversity that result by the use of several nodes, the MISIMA multi-node combining, that results from the weighed summation of the outputs of each node, reduces the ISI. Those predictions have been illustrated on high rate underwater communication real data for which multi-node processing resulted in sensible gains in both MSE and BER, namely when environmental stationarity can be assumed. Also, an interesting 'at least equal to the best' behavior of the multi-node combining has been observed. Depict MISIMA was developed for a network of nodes running a pTR equalizer it can be readily applied to any network where nodes are running other equalizers as a DFE, FSE, etc. In such context the main advantage of MISIMA relays in its ability to preserves the efficiency of the network as one node encounters severe difficulties as it happens e.g. in a pTR equalizer when the IRs estimate by the probe loss validity or in a DFE equalizer when due to numerical errors the equalizer starts to diverge. However, in a non stationary environment, multi-node weighted combining stems from the ability to continuously adapt the weighted coefficients to channel variations. Considering that a "physical-model" can be extracted from the closed form of the coefficients (11) a Kalman filter approach is suggested for future work.

The MISIMA use of a single coefficient per node resembles the behavior or the pTR based Unconstrained Minimum MSE (UMMSE) equalizer proposed by Gomes et al. [5], where in order to optimize the MSE with a pTR based equalizer a single coefficient per hydrophone equalizer is adopted. Since no "physical-model" was considered in the UMMSE development a Winner filer strategy was considered for the coefficients adaptation. Since in digital communications MSE and ISI are closed related it is proposed as future work to integrate MISIMA in to UMMSE in order to consider a Kalman filter strategy for the coefficients adaptation.

\section{ACKNOWLEDGMENTS}

The authors would like to thank the NATO Undersea Research Centre (NURC) for the loan of the acoustic sound source and the support of the NRP D. Carlos I crew during the RADAR'07 sea trial. This work was financed by FCT, Portugal, under UAB project (POCI/MAR/59008/2004), RADAR project (POCTI/CTA/47719/2002), and PHITOM project (PTDC/EEA-TEL/71263/2006).

\section{REFERENCES}

[1] R.D. Jackson and R.D. Dowling. Phase conjugation in underwater acoustics. J. Acoust. Soc. Am., 89(1):171-181, January 1991.

[2] W.A. Kuperman, W. Hodgkiss, H. Chun Song, T. Akal, C. Ferla, and D. Jackson. Phase conjugation in the ocean: Experimental demonstration of an acoustic time-reversal mirror. J. Acoust. Soc. Am., 103(1):25-40, January 1998. 
[3] A. Silva, S. Jesus, J. Gomes, and V. Barroso. Underwater acoustic communications using a 'virtual' electronic time-reversal mirror approach. In P. Chevret and M.Zakharia, editors, 5th European Conference on Underwater Acoustics, pages 531-536, Lyon, France, June 2000.

[4] R.D. Dowling. Acoustic pulse compression using passive phaseconjugate processing. J. Acoust. Soc. America, 95(3):1450-1458, 1994

[5] J. Gomes, A. Silva, and S.M. Jesus. Adaptive spatial combining for passive time-reversed communications. J. Acoust. Soc. America, 124(2):1038-1053, August 2008.

[6] A. Silva, S. Jesus, and J. Gomes. Passive time reversal probe-signal capture optimization for underwater communications. In Proc. of the UAM'07, Heraklion, Crete, Greece, June 2007.

[7] A. Silva, F. Zabel, and C. Martins. Acoustic oceanographic buoy telemetry system. Sea Technology, 47(9), September 2006.

[8] C. Soares and S.M. Jesus. Real-time environmental inversion using a network of light receiving systems. In Proc. of the Martech Conference, Barcelona, Spain, November 2007.

[9] S.M. Jesus, J.-P Hermand, and J.-C LeGac. A buoy network system for acoustic monitoring. In Rapid Environmental Assessment Conference, La Spesia, Italy, September 2007.

[10] S.M. Jesus, C. Soares, and N. Martins. Water column tomographic inversion with a network of drifting buoys. In Proc. ACOUSTICS'08 Conference, Paris, France, June 2007.

[11] J.G. Proakis. Digital Communications. McGraw-Hill, Massachusetts, 1995. 